\title{
Autoclaves for Aerospace Applications: Issues and Challenges
}

\author{
A. R. Upadhya, ${ }^{1}$ G. N. Dayananda, ${ }^{2}$ G. M. Kamalakannan, ${ }^{2}$ J. Ramaswamy Setty, ${ }^{2}$ \\ and J. Christopher Daniel ${ }^{3}$ \\ ${ }^{1}$ Council of Scientific and Industerial Research National Aerospace Laboratories (CSIR-NAL), Bangalore 560017, India \\ ${ }^{2}$ Centre for Societal Missions and Special Technologies, CSIR-NAL, Bangalore 560017, India \\ ${ }^{3}$ Advanced Composites Division, CSIR-NAL, Bangalore 560017, India
}

Correspondence should be addressed to J. Ramaswamy Setty, ramaswamy@nal.res.in

Received 15 February 2011; Accepted 6 April 2011

Academic Editor: R. Ganguli

Copyright ( 2011 A. R. Upadhya et al. This is an open access article distributed under the Creative Commons Attribution License, which permits unrestricted use, distribution, and reproduction in any medium, provided the original work is properly cited.

\begin{abstract}
The Council of Scientific and Industerial Research National Aerospace Laboratories (CSIR-NAL), Bangalore, India has been engaged in the research of autoclaves for the past three decades and has pioneered their development and usage in India for aerospace/aircraft structural applications. The autoclaves at CSIR-NAL have played a significant role in all the major national aircraft/aerospace programs. The largest aerospace autoclave in India (working size of $4.4 \mathrm{~m}$ diameter and $9.0 \mathrm{~m}$ length) has been successfully commissioned at CSIR-NAL. This paper gives the technological challenges faced and the innovative concepts that were introduced in these autoclaves.
\end{abstract}

\section{Introduction}

Autoclaves have become indispensable tools/equipments for processing high quality polymer composite aerospace/aircraft structural components [1]. Today, in the aircraft industry, investments in these equipments are regarded to be strategically important. Autoclaves are now being used to produce very large aircraft components such as wing and fuselage. They can process a wide variety of materials, including thermoset [2] and thermoplastic [3] based composite aircraft parts with varying contours and complex shapes. Thermosets are widely used as they are less expensive compared to thermoplastics.

The quality requirements of the present aircraft industry are indeed most stringent. In addition, there is a dire need to improve the efficiency and cost effectiveness of aircraft structural systems apart from ensuring reliable and consistent processing methods. In such a scenario, it is imperative that the autoclave designer properly takes into account the various governing criteria involved in processing and developing state-of-the-art autoclave systems that satisfactorily address the diverse and complex requirements stated above. The typical vacuum bagging scheme adopted in the autoclave molding technique for a composite component along with several consumables processed is shown in Figure 1, and a typical cure cycle for a thermo set epoxy resin is shown in Figure 2. In addition to handling a wide variety of consumables, modern autoclaves must incorporate adequate safeties [4] and ensure minimum maintenance costs.

The design of these complex systems is multidisciplinary in nature and encompasses mechanical, process control, and instrumentation engineering. Invariably, the state-of-the-art autoclave systems are completely automated with reliable computer control systems.

The computer of these modern autoclaves is required to execute the selected cure cycle, by sequentially starting various subsystems, download set values at regular time intervals to the front-end controllers, acquire, store, and archive the data, monitor cure status and faults, generate alarms, and perform the functions of sequential shut down and reporting [5]. Ease of maintenance, fail-safe operation and reliability are among the key drivers in modern autoclaves. Low cost of ownership also needs to be considered in today's context.

In situations where more autoclaves are present at a single site, the present trend is to interconnect these systems through Local Area Networks (LANs), so that the overall efficiency of the network/group of autoclaves is enhanced, and the utilization of the autoclaves is optimized. The system engineering of these complex systems necessitates that several issues associated with them are properly addressed. 


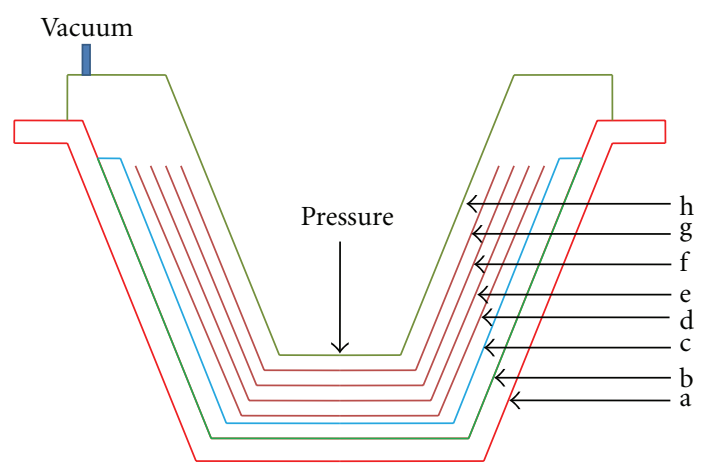
a: Mold
e: Porous release film
b: Release agent applied to mould
f: Pressure pad
c: Layup
g: Breather
d: Bleeder
h: Bag

FIGURE 1: Typical Autoclave-molding bagging scheme.

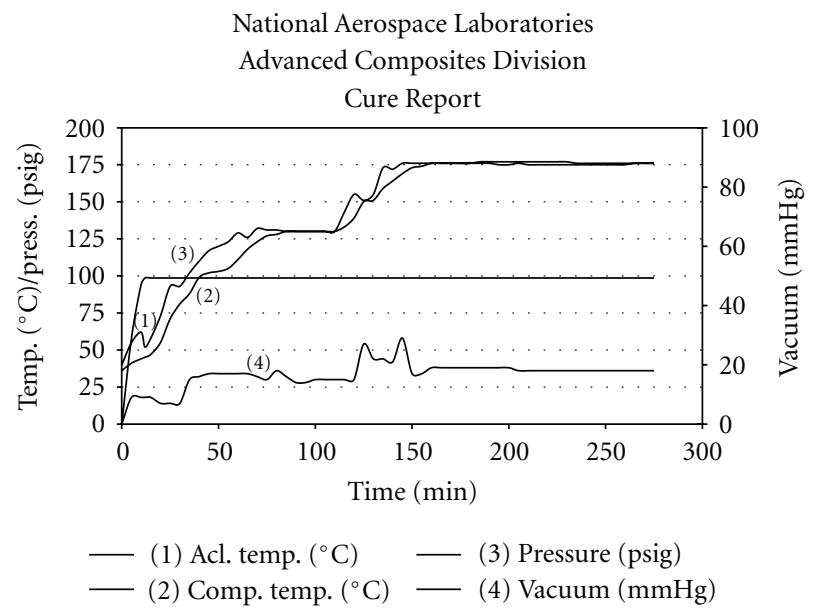

FIgURE 2: Typical cure cycle for a thermo set epoxy resin.

In the process, it is natural that the design and engineering of these systems pose innumerable challenges. The autoclave operating parameters such as temperature and pressure are based on the resin systems used. Generally, the epoxy resins require temperatures within $200^{\circ} \mathrm{C}$ and pressures of $7 \mathrm{bar}(\mathrm{g})$. The service temperatures of these epoxy resin-based structural components are restricted to about $120^{\circ} \mathrm{C}$. Extensive literature is available which details the processing of thermo set composites using autoclaves $[1,6]$. The present authors have discussed various issues involved in the development of autoclave technologies [7]. As already mentioned, the large size of these structural components (wing and fuselage) has created the need for very large size autoclaves (typically 6 to 10 meters in length and 3 to 5 meters in diameter) in the industry. A very large state-of-the-art aerospace autoclave with computer control is shown in Figure 3. Figure 4 shows the mimic of the control panel of this autoclave.

Nowadays, in the process of replacing more and more metallic parts by composites (in zones of aircraft that encounters higher temperatures such as bypass duct and cowling plates of military aircraft), there is an increasing demand to enhance the service temperatures of polymer composite structural components to about $200^{\circ} \mathrm{C}$ from the present $100^{\circ} \mathrm{C}$ or so. This means higher curing temperature and pressure in the order of 300 to $350^{\circ} \mathrm{C}$ and up to $15 \operatorname{bar}(\mathrm{g})$, respectively. Resin systems such as phenolics, bismaldehydes, and other thermoplastics fall under this category. This necessitates the development of high temperature/high pressure autoclave systems. The development of large autoclave systems presents itself with one set of challenges such as handling of massive door, quick locking door system, temperature uniformity, special material procurements for door and shell flanges, fabrication, transportation, and so forth. The development of high-pressure high-temperature (special operating conditions) autoclaves, on the other hand, presents itself with a completely different set of problems such as sealing at high temperatures, thermal stresses, blower shaft sealing systems, robust sensing and measurement systems that can withstand the higher operating conditions, and so forth. The issues and challenges associated in the analysis, design, fabrication, testing, assembly, and transportation of large and special autoclaves are presented here, system-wise.

\section{Autoclave's Main Shell with Quick-Lock Door}

The main shell is a sealed container and provides the working space for the components to be processed under pressure, temperature, and vacuum. A quick lock door is a feature of all modern autoclaves. The quick lock door comprises toothed flanges and locking mechanisms. For large autoclaves, the design of the door handling system is a challenge. A good design should occupy the least space for movement of the door and have flexibility aligning the door with the shell. The material for the shell is boiler-quality steels such as SA516 Gr. 70. These steels have high yield stresses even at elevated temperatures (up to $350^{\circ} \mathrm{C}$ ). The material for the flanges of large autoclaves is usually forged steel such as SA266 Gr. 2. Forgings possess uniform grain structure, superior strength, and impact properties and have improved machinability characteristics. Very few companies in the world can make forgings as large as 5 -meter diameter in a single piece. If forging a large single piece is by itself a daunting task, machining the forging to cut the required teeth on the flanges, to close tolerances, to ensure locking is an equally challenging task. Floating lock rings, which have been used in a large number of autoclaves, comprise of mating teeth, and together with the door flange teeth ensure locking of the autoclave. The teeth are cut in either horizontal or vertical machining centers. Nowadays, it is common to find the shell flange along with lock ring being integrally welded to the main shell, even for large vessels. This substantially reduces the weight of the door locking system as it not only eliminates the massive locking ring but also the associated support system to keep the lock ring floating. This design will reduce the weight of the door system by as much as $30 \%$. The doors of large autoclaves need to be rotated by about $90^{\circ}$ for loading the component or mould for cure. The large autoclave doors due to their large mass have to be rotated with extreme care to minimize the inertial loads. Further, a good autoclave 


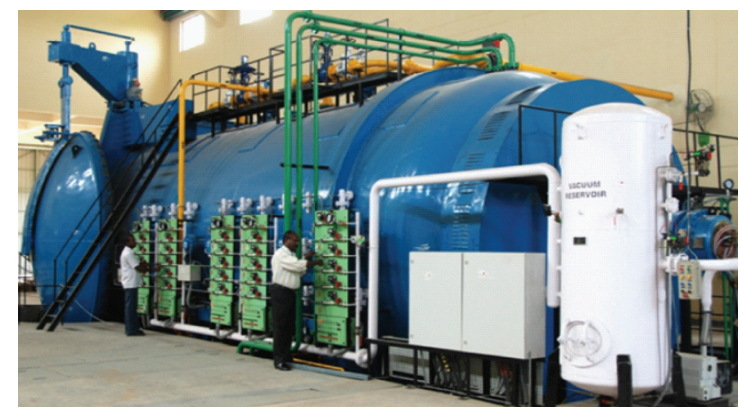

FIGURE 3: India's largest aerospace autoclave with working space of $4.4 \mathrm{~m}$ dia $\times 9 \mathrm{~m}$ length at CSIR-NAL, Bangalore.

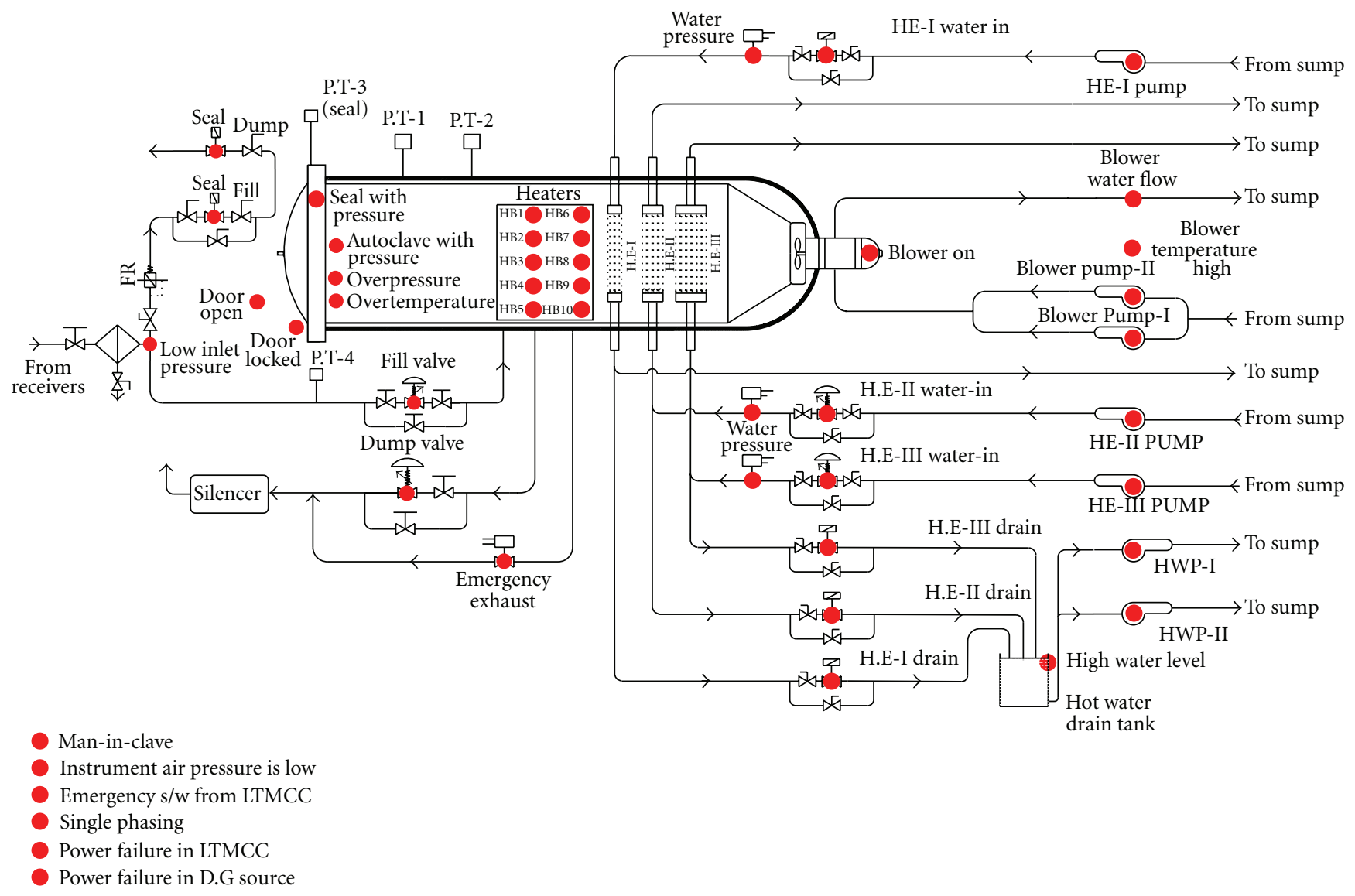

Figure 4: Mimic of the Autoclave.

design ensures the minimum use of precious layout space for opening and parking of the door [7]. The door should open in the beginning along the longitudinal axis of the autoclave in order to avoid interference with the lock ring and then be guided/steered in a predetermined path, such that it traverses the least possible distance and occupies the least possible space both in front of the autoclave as well as when it is tucked to the side for parking. Davit arms are popularly employed to handle large doors. A good door-steering mechanism design will necessarily exploit CAD modeling to carry out simulation studies. The locking of the door for pressure tightness is done by rotating a floating lock ring as already stated. However, in many large autoclave designs, the locking is also ensured by rotating the door in a fixed-shell flange. This type of design ensures a more accurate alignment of shell and door and improves the sealing performance. As per ASME pressure vessel code, it is mandatory for pressure vessels with quick closing doors to have a positive door-lock safety device, which should prevent the door operation when the shell is pressurized. The modern autoclaves have foolproof door-lock safety device, which makes it almost impossible to operate the door when the shell is under pressure.

During curing of composites, the customer usually stipulates that the outside skin temperature of the autoclave shell should not generally exceed $25^{\circ} \mathrm{C}$ above the ambient. This necessitates providing adequate insulation lining in the 


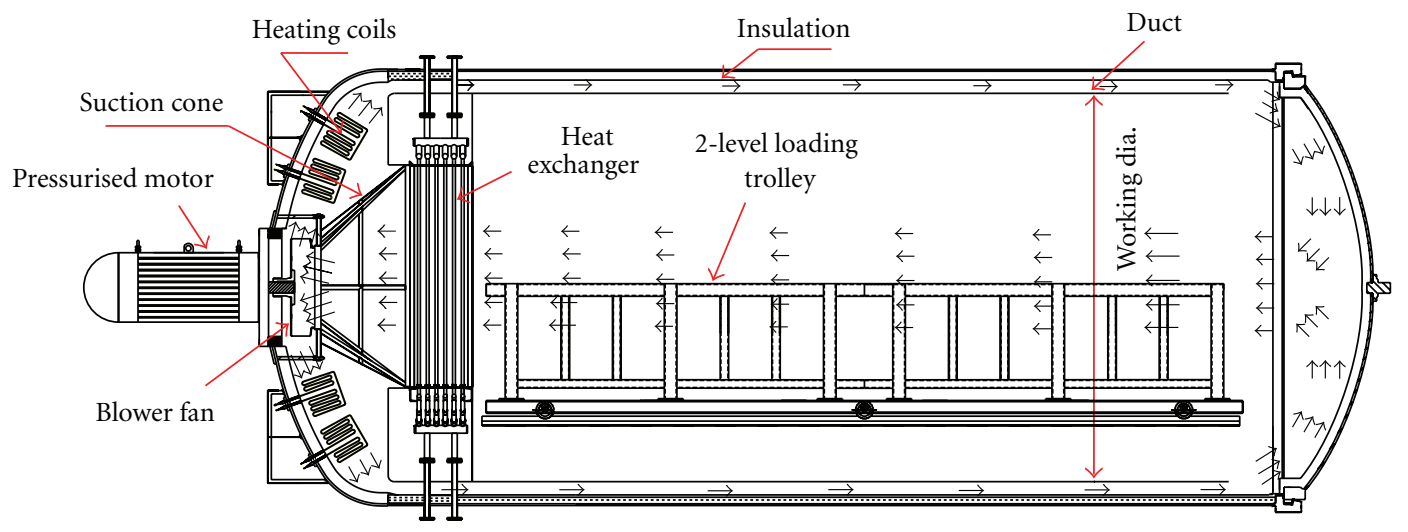

FIGURE 5: Schematic of air circulation and heating system.

interior of the vessel. Mineral wool is generally the material of choice.

Sealing the pressure vessel is another issue for large autoclaves. Either the inflatable seal or the lip seal is used. The inflatable seal expands and seals against the door flange whereas the lip seal deforms and presses against the door flange to seal the pressure. The inflatable seals have an annular groove in which the pressure is usually higher than the autoclave operating pressure. The concentricity and the dimensional accuracy of the groove, the overall dimensional accuracy of the seal, and the annular metal seating in the flange are the key parameters that ensure leak-tight sealing of the autoclave. The inflatable seal does not demand very precise alignment between the flanges as it can expand under pressure and fill the nonuniform gaps. The power required to lock the door is also less as the seal is not in contact with the flange during locking. This is the most preferred choice for large autoclaves. On the other hand, the lip seal requires precise alignment and uniform gap, and it needs to be pressed during locking. This requires more power for locking.

\section{Air Circulation System}

Forced gas (nitrogen or air) circulation systems are commonly used in autoclaves. The schematic is shown in Figure 5. The circulation system consists of a centrifugal blower and ducting. The heaters are placed around the impeller. The centrifugal blower takes in gas axially and discharges it radially. The gas that is discharged from the impeller passes over the heating elements. The blower fan is adequately sized to ensure a velocity of $1-2 \mathrm{~m} / \mathrm{sec}$ on the component at ambient conditions. The gas circulation unit also performs the task of accelerating the cooling process by removing the gas on the outside surface of the cooling tubes at a faster rate. Modern autoclaves have a flangemounted blower motor, which is encased in a pressure-tight casing and connected to the rear of the autoclave. Thus, the motor rotor, stator, and the mechanical components such as the bearings directly encounter the autoclave pressure. Such blower arrangements are generally water-cooled to ensure that the motor windings and bearings are not subject to higher temperatures. The power rating of the blower motor of a large autoclave can be in the range of 100 to $150 \mathrm{~kW}$.

\section{Heating System}

Autoclaves are heated either electrically or by indirect gas firing (circulating externally heated or cooled thermic fluid). Electrical heating that gives precise control of autoclave ambient temperature is cleaner and more amenable to advanced computer controls. Modern autoclaves use SCR drives, which form part of the closed-loop heating-control systems and can provide very fine control of the heaters. The electrical heating capacity is based on the charge and the resin system requirements of the cure cycle. Typically, a 4.5-m diameter $\times 9$-m length autoclave requires an installed heater capacity of about a mega Watt. Normally a number of heater elements (typically of ratings in the range of 5$10 \mathrm{~kW}$ ) are grouped in banks and connected in the star or the delta configuration. The heating elements are made of nichrome/kanthal filament, magnesium oxide insulator with an outer sheath of incoloy or steel.

\section{Cooling System}

This system is meant for cooling the autoclave ambient. The processing of composites requires variable cooling rates as demanded by the resin system. In electrically heated autoclaves, the heating rate is controlled by either switching off the heater banks or by varying the heater-input power. The control of cooling system is more involved as there are a number of variables that affect the cooling. They are temperature difference between the autoclave ambient and cooling medium, rate of flow of cooling medium, heat transfer area, heat transfer coefficient of the cooling coil which is again a function of type of flow, that is, cross flow or parallel flow, conductivity of cooling coil material, and velocity of autoclave medium across the Heat Exchanger (HE). Generally, the cooling rate is controlled by varying the flow of cooling water. In some autoclaves, both air and water are used as a cooling medium. The modern autoclaves control all the three parameters, that is, rate of flow of cooling medium, temperature of cooling medium, and heat transfer 
area. Though this method is expensive, it is justified in large as well as high-temperature autoclaves where there is a wide range of loads to be cooled. To save the water, a closed-loop cooling system is generally employed. It consists of HE coils inside the autoclave, control valves, draining system, cooling tower, cooling water pumps and water treatment plant, and so forth. In the design of cooling system, the major challenge is to fast and effectively drain the cooling medium from the autoclave HE (Figure 6). Any delay in draining water from the HE not only results in draining away the heat of the autoclave during heating phase but also in formation of steam inside the HE which could damage the HE tube, if the steam pressure exceeds a certain value. Several methods are employed for the automatic draining of the cooling medium. Simple way of draining is to create the least resistant path for the water to flow. That is, provide a sump just below the autoclave heat exchanger and then pump back the water to the cooling water sump. This also reduces water wastage and prevents hot water/steam entering the cooling tower.

The modern autoclaves employ seamless stainless steel (SS) tubes with extruded fins on the tube, which minimize pressure losses. These SS tubes have high heat-transfer area per unit volume of space they occupy.

\section{Pressurization System}

The system must ensure that the required pressurization rates in the autoclave are met. The average pressurization rate in modern autoclaves is $2 \mathrm{bar} / \mathrm{min}$. Nowadays, many autoclaves use nitrogen as the pressurization medium instead of air. This is because the autoclave cure consumables are highly inflammable in the air medium due to the presence of oxygen. There have been several reports of autoclave fire resulting invariably in the loss of the component. Though the nitrogen medium ensures fire-free autoclave cure cycles, care must be taken to avoid danger to personnel (possibility of asphyxiation) in nitrogen environments due to the lower oxygen levels.

The nitrogen gas pressurization system consists of a primary compressor, nitrogen plant, booster compressor, storage tanks, and associated piping circuitry as shown in Figure 7. The primary compressor takes in the air from the atmosphere and pressurizes it to $7 \mathrm{bar}(\mathrm{g})$. The nitrogen plant receives the air at $7 \mathrm{bar}(\mathrm{g})$ and by a process known as Pressure Swing Adsorption (PSA) isolates nitrogen from the atmospheric air. The PSA has emerged as one of the popular methods of producing nitrogen. The nitrogen purity produced by this method is of the order of $99 \%$, which is adequate for curing of aerospace polymeric composites in autoclaves. The nitrogen, thus, isolated is further pressurized using a booster compressor to higher pressures, typically 17 22 bar. Higher pressure is required to create sufficient pressure differential in order to meet the required pressurization rate. The nitrogen storage tanks are sized in such a way that the free-air delivery (FAD) of these storage tanks is 2.5 times the FAD of the autoclave.

For example, the FAD of a 200-cubic-meter autoclave operating at $7 \mathrm{bar}(\mathrm{g})$ would be 1400 cubic meters and consequently, the FAD of the nitrogen storage tanks would

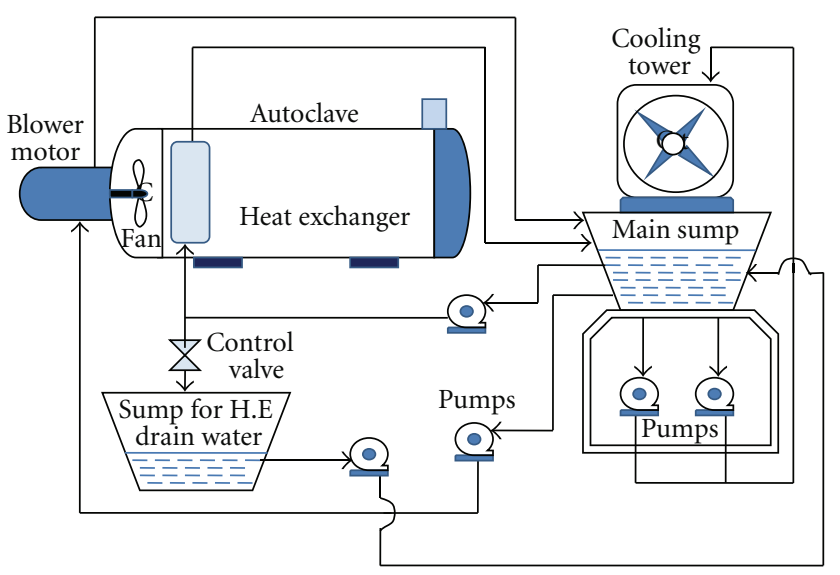

FIgURE 6: Schematic of cooling system.

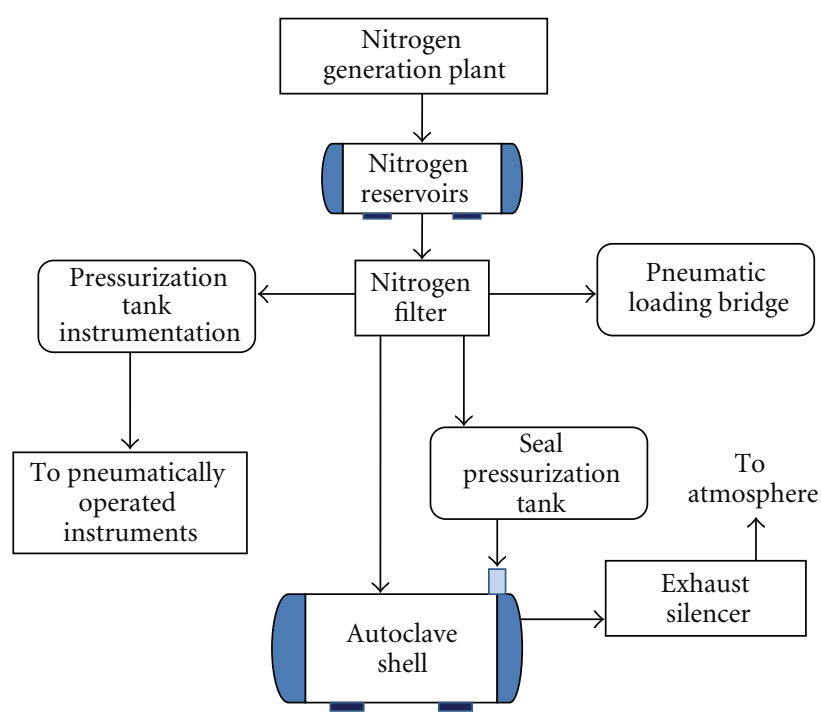

Figure 7: Schematic of pressurization system.

be 3500 cubic meters. The material used for the storage tanks is boiler-quality steel.

\section{Vacuum System}

An advanced vacuum system is an essential ingredient of modern autoclaves. The system consists of vacuum pumps, vacuum reservoirs, buffer tanks, measurement lines, and suction lines as shown in Figure 8. The measurement, suction and vent lines are part of the closed-loop vacuum control that ensures the required level of vacuum inside the bag and on the component. A good autoclave design must provide for adequate number of vacuum ports. It must also provide for maintaining different levels of vacuum in different bags at the same time. Bag leaks during cure are not uncommon. Therefore, the pumps and the vacuum reservoirs must have adequate buffer capacity. A guideline for the selection of the pump is to have a pump of capacity 7 cubic meter/hr for a bagging area of $1 \mathrm{~m}^{2}$. Likewise, the conductance of the vacuum lines must be adequate. Typically, a $4.5 \mathrm{~m} \times 9 \mathrm{~m}$ 


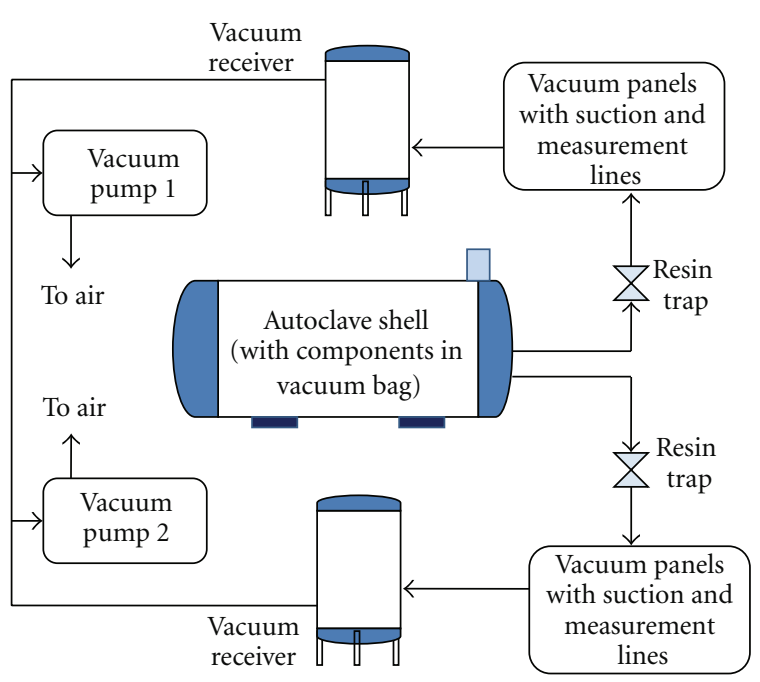

FIGURE 8: Schematic of vacuum system.

autoclave could have as many as 60 measurement and suction lines. The corresponding pump capacities are typically about $180 \mathrm{~m}^{3} / \mathrm{hr}$, and the reservoir capacity is of $6 \mathrm{~m}^{3}$. The range of vacuum required in the bag is 2-500 torr depending on the curing systems. In addition, modern designs incorporate advanced vacuum-sensing and failure warning/protection systems. Besides, the resin traps must serve the purpose of trapping the excess resin that is squeezed out during cure and ensure proper drain out.

\section{Loading System}

The loading system of an autoclave consists of a loading platform for positioning the components/moulds to be cured. Based on the diameter of the autoclave to enable loading of more number of components, two-tier/three-tier loading is being employed. A loading crab is employed to push and pull the platform from the autoclave. To enable easy loading of components, it is a general practice to install the autoclaves in a pit, so that the top surface of the loading platform is flush with the floor. In this case, it requires a loading bridge to be deployed to bridge the gap between autoclave door and pit. Several designs are employed for the bridge. The tilting bridge and scissors-type bridge are common designs. Generally, these bridges use pneumatic pressure available at the autoclave as a prime mover.

\section{Electrical System}

Electrical systems of large autoclaves have to handle high currents at high voltages. Hence, their safe operation is of paramount importance. They should also be very reliable to ensure proper manufacture of expensive composites amidst failure of one or more subcomponents. To maintain reliability without compromising safety, sufficient redundancy and localized "trip" circuits should be built in the system. Further, the electrical system should provide necessary feedback signals to the control system and respond to various commands from it. An added feature is the operation of all the electrical motors and heaters of the autoclave through manual mode, which helps in saving the composites being cured against failure of control system.

The electrical panel can be modular with separate cabin, ON/OFF pushbuttons, lamps, isolator, and so forth, for every electrical machine. This would provide added convenience to operator during operation and maintenance. Each lowpower motor (less than $10 \mathrm{~kW}$ ) should have independent protection against short circuit, overload and earth faults. High power AC motor such as Blower Motor should be operated either through Star/Delta Starter or through an AC drive. Operating the blower through an AC drive, though expensive, offers the number of advantages given below:

(i) the rotational speed can be reduced as the autoclave pressure increases, in order to keep the mass flow constant,

(ii) since the blower runs at lower speed at higher pressure, its power rating reduces,

(iii) soft starting, smooth turn-off, better operating current limits, interlocks, and so forth. increases the motor life.

CSIR-NAL's large autoclave is built with both Star/Delta and AC-drive operations, so that even in case of AC drive fault, blower can be operated. Another important component of the electrical system is the heater-power controller. As a cost-effective and novel feature, $20 \%$ of heater is operated through power controller, and the balance $80 \%$ is operated through discrete controllers (contactors) without sacrificing the required control accuracy [8].

\section{Control and Instrumentation (C\&I) System}

Autoclave Control and Instrumentation (C\&I) system plays a very important role in ensuring reliable processing or curing of composite structures [4]. These systems are quite complex and require well-trained engineers to design, build, troubleshoot, and upgrade. As the electronics and software system technologies continues to grow, the C\&I products continue to change for better, and the older products soon become obsolete. Typically, C\&I system needs to be upgraded or completely replaced every ten years. However, the benefits accrued in terms of flawless composite manufacture justify such replacement.

With system reliability being the key issue, during design phase, it is assumed that every individual product would fail at one point of time or the other, and yet the operator should be able to continue the process. As a measure of abundant caution, autoclaves are built with redundant computers, serial port servers, power supplies, sensors, and so forth, so that the system will be operable even if one or more components fail. In addition to redundancy on the component level, multiple modes of operations, namely, automode, semi-auto mode and manual modes are provided.

In auto mode of execution, operator can choose the required cure cycle (or program a new one) and command the system to execute it. In the event of computer failure, 


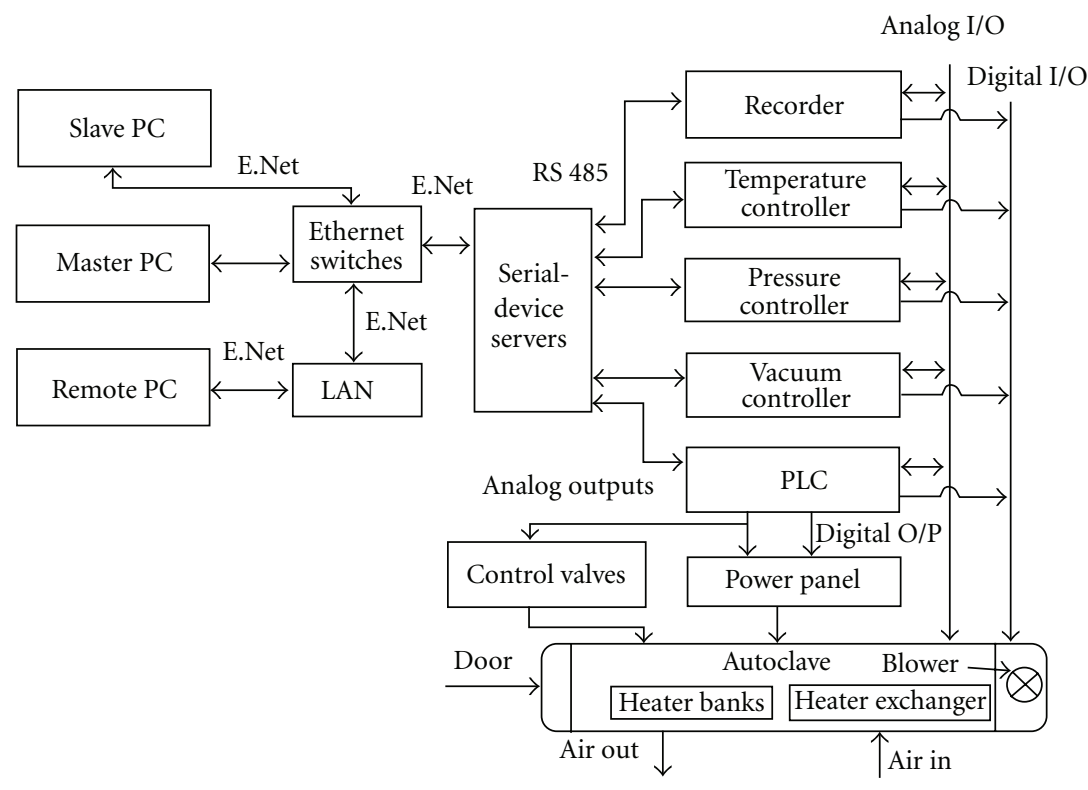

Analog I/Os: Analog inputs and outputs

Digital I/Os: Digital inputs and outputs

E.Net: Ethernet

Figure 9: Architecture of the control system.

which can occur at any time during the cure without a warning, the semi-auto mode provides a very useful fallback arrangement. In this mode, operator can feed the set points for temperature, pressure, and vacuum directly to the respective controller and initiate the segments of the cure cycle such as heat, pressure, and vacuum cycle. Figure 9 shows the Architecture of the control system developed for the large autoclave.

The control system consists of programmable PID (proportional-integral-derivative) controllers for effecting the closed-loop control of temperature, pressure, and vacuum; Recorders for the display, plot, and storage of all the analog input signals such as part temperature at different locations, pressure, vacuum at different parts, and so forth; PLC (programmable logic controller) for ensuring safety, interlocks, sequential operation, status/alarm display, and so forth. All these components are connected to a pair of serial device servers (for redundancy) and in turn accessed by the computers through Ethernet links. The communication links used in the autoclave system include RS485, USB, and ethernet [9]. Design drivers of the control system as already stated are:

(i) reliability and redundancy,

(ii) fail Safe,

(iii) ease of operation and maintenance.

\section{Cure Control Software}

Microsoft Visual Studio is one of the popular platforms for the autoclave software development. The software performs the general functions of SCADA (supervisory control and data acquisition), in addition to carrying out jobtemperature control. Protocols and tools used in the software include MODBUS, TCP-IP, OPC (OLE for process control) Client-Server routines, data integrity check through CRC (cyclic redundancy check), and so forth. Another unique feature is the automatic takeover of control by the slave computer in case of problem in the master computer. This is achieved by the automatic tracking of the user-specified cure cycle, control parameters, and the current state of the process through the slave computer.

One of the important features of the software is the capability to control the part-temperature as per user requirement. The user can specify in real time, the type of required part temperature control, which could be based on the leading sensor, lagging sensor, or the average of a group of selected sensors. The user can also have multiple groups and different control sensor choices for each group. For example, temperature of part no. 1 can be based on the leading sensor among a set of sensors, while temperature of part no. 2 can be based on the lagging sensor. The algorithm predicts the expected change in the part temperature based on its past behavior in that cure and effects the required changes in control of the air/gas temperature.

\section{High-Temperature, High-Pressure Autoclaves}

As already mentioned, there is a concerted effort to move towards higher temperature and higher pressure curing systems in order to address the manufacture of airframe components that need to withstand higher service temperature. Bismaldehydes and phenolic resins are being considered 
for this purpose. This has necessitated the development of autoclaves that operate at temperature beyond $350^{\circ} \mathrm{C}$ and pressure as high as $15 \mathrm{bar}(\mathrm{g})$. High-quality boiler-grade steels, such as SA516 Gr. 70 or other equivalents that have high-yield stresses even at temperature as high as $350^{\circ} \mathrm{C}$ are used for the main shell of these autoclaves. At such high temperature, sealing the autoclave main door is a challenge because they experience the thermo-mechanical loading. Elastomeric sealing materials which can withstand up to $350^{\circ} \mathrm{C}$, for continuous exposure are not available. High-temperature sealing materials like viton, silicon can withstand temperature up to $250^{\circ} \mathrm{C}$ only. To make them adaptable to these applications, the sealing materials need to be properly protected by baffles or by deflectors such that they do not directly get exposed to hot autoclave ambient. In addition, the feed throughs for the heating elements as well as for the various temperature- and pressuresensing elements have to be properly sealed, such that there is no pressure leak. It is also important to ensure that though the autoclave ambient is beyond $350^{\circ} \mathrm{C}$, the outside skin temperature does not exceed $25^{\circ} \mathrm{C}$ above the ambient. This calls for proper design of the insulation system. Mineral wool of adequate density is generally used for this purpose. Likewise, the loading trolley system that gets into the autoclave has to be designed to withstand the extreme autoclave operating conditions (simultaneous thermomechanical loading). Conventional bronze and axle, or bearing bush cannot be used in the trolley wheels. We need to have higher clearances between two mating surfaces and use graphite-based solid lubricants in the bearings. The charge additionally loads the loading system, which places an extra demand on the design. The pressurized blower system for the high-pressure, high-temperature autoclave, which is a feature of modern autoclave, is another area of great challenge for the designer. It is important to ensure that the motor windings do not experience the autoclave conditions and are within the prescribed temperature limits. The mechanical drive consisting of the impeller and drive shaft must withstand the high-operating conditions. The bearings on the driveshaft must be adequately cooled such that the temperature near the bearings does not exceed $120^{\circ} \mathrm{C}$. The design must ensure that the structural integrity of the overall system at the extreme operating conditions remains intact. Adequate clearances need to be given in the ducting and piping to take care of thermal expansions. The heat exchanger piping is generally provided with bellows to reduce thermal stresses. The design of bellows poses a challenge as they need to withstand high temperatures, thermal shocks, and high pressures. Further, the hightemperature resin system (bismaldehydes and phenolics) polymerization reactions are exothermic to a far higher degree than the conventional epoxy resin systems. Therefore, the closed-loop temperature control system must be versatile enough to prevent temperature overshoots during cure. In addition, the exothermic reactions from these resin systems release a much higher quantity of toxic wastes, compared to conventional resin systems. Some of the resin systems give out water as a byproduct during the crosslinking process (curing). Therefore, the vacuum system must be engineered

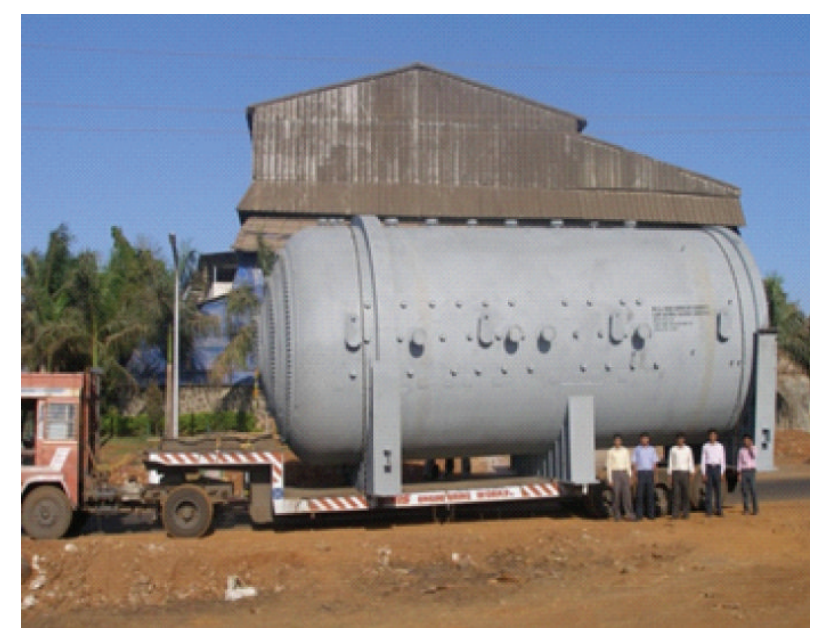

FIGURE 10: Transportation of the autoclave shell.

to cope with the larger quantum of toxic substance release and for effective removal of water.

\section{Transportation}

Smaller autoclaves up to about 1 meter in diameter are integrated with all the subsystems and transported after complete system checks. However, for larger autoclaves, normally the shell and other subsystems are transported separately, and the system integration and commissioning are carried out at site. Very large autoclave shells of typically 5 to 6 meter diameter and 12-15 meter length (outside dimensions) have to be transported with extreme care, particularly in developing countries where the infrastructure may not be supportive for such large systems (Figure 10). The load-carrying capacity of the roads, the clearance in terms of height of the bridges and arches and the overhead high-voltage power lines have to be carefully examined before chalking out the route for transportation of these huge systems.

Wherever the infrastructure does not favour the transportation of these systems either by road or on the water, it is advisable to build them in situ.

\section{Installation, Integration, and Commissioning}

Installation of the autoclave shell includes removing of the shell from the trailer and positioning it on the foundation. Whenever the autoclave is installed in a pit, lowering of the shell is achieved through a carefully articulated series of movements of the shell using a combination of stackedup wooden logs and chain pulley blocks, which is shown in Figure 11. During the final lowering extreme, care should be taken such that the front saddle, which was designed to be the fixed saddle, gets properly located and is securely held to the ground by means of mechanical fasteners. The rear saddle is a floating one, which is simply resting on the foundation to allow for thermal expansions/contractions.

Amongst other issues, an important challenge is in arriving at the appropriate layout. All the subsystems need 


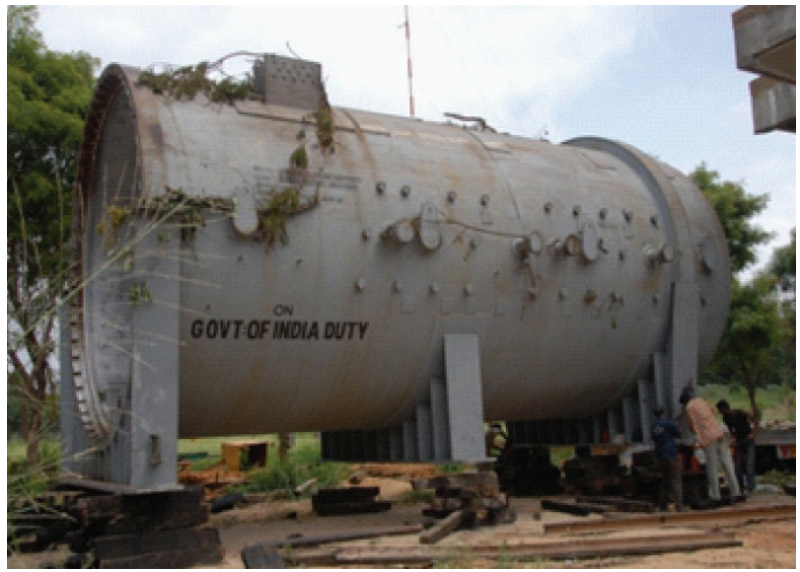

FIGURE 11: Erection of the shell at the site.

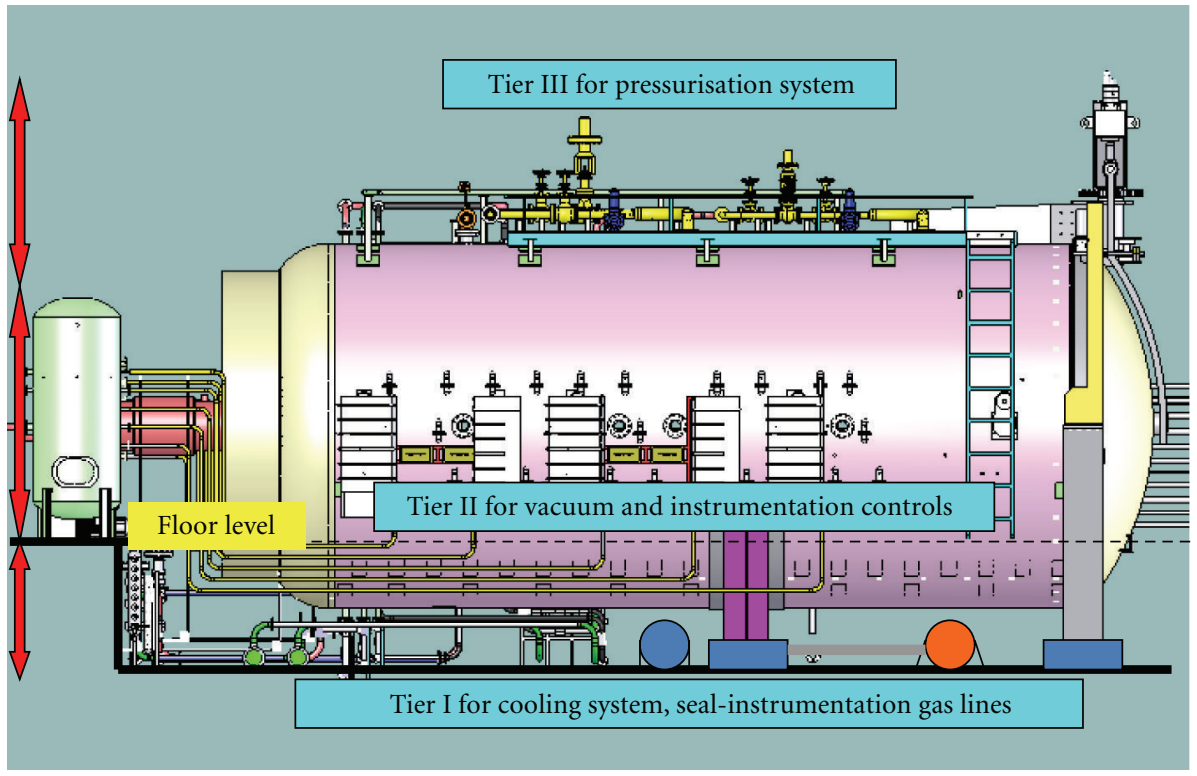

Figure 12: Three-tier layout of subsystems.

to be properly installed without compromising on the accessibility and maintenance aspects, consuming least shop floor space while at the same time maintaining good aesthetic appearance. The site conditions also influences the layout. The layout and pipe routing is particularly more complicated for large autoclave systems where more number of vacuum lines and other associated systems are involved. One should follow the standard industrial piping practices and provide sufficient flange joints for ease of assembly and maintenance. Pipelines need proper support in order to prevent thermal and other loading of autoclave ports. Provision must be made to accommodate thermal expansions in the pipelines. Hot gas exhaust and hot water pipes should have thermal insulation cover and are to be erected with more care.

Apart from the large number of accessories, one should accommodate several power cables and instrumentation wires, a number of processpipelines and associated hardware.
In general, the power panel should be located close to the autoclave, particularly to the heater and blower terminations to bring down the cable lengths. At the same time, it should be close to the main power supply feeder, as the cost of bus ducts (insulated electrical conductors that feed low-tension voltage and high currents) is very high. Control panel should also be located close to the autoclave, particularly to the sensor terminations to minimize the electrical noise effects. It is also important to segregate and route the AC (Alternating Current) and DC cables separately.

An innovative three-tier layout was conceived to accommodate all these systems for the large autoclave. It was configured using CAD tools such as SOLIDWORKS. Figure 12 shows the three-tier configuration, which is ideal for medium- to large-size autoclaves.

Cooling water lines, instrumentation air lines, seal pressurization lines, and other gas processing units, which do 


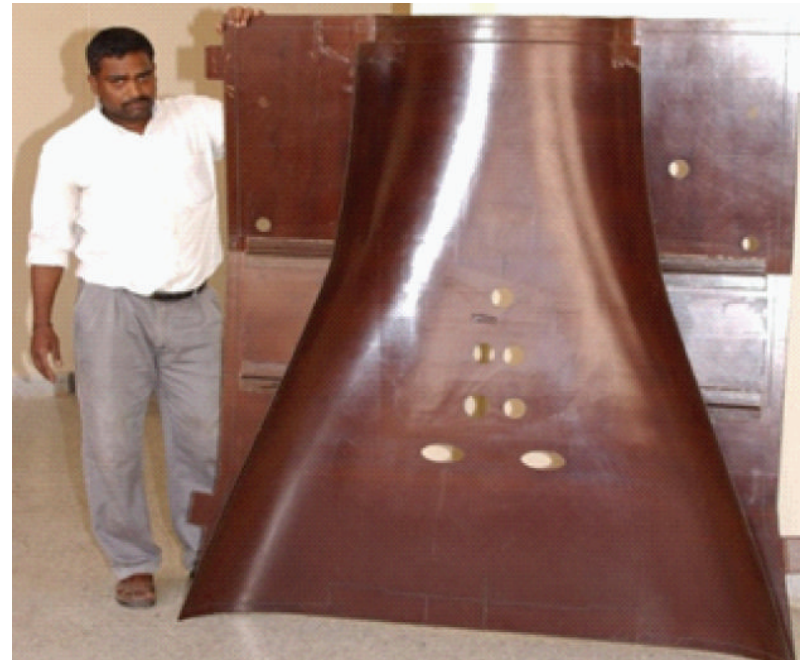

Figure 13: Trouser duct of LCA Aircraft cured in an autoclave at CSIR-NAL.

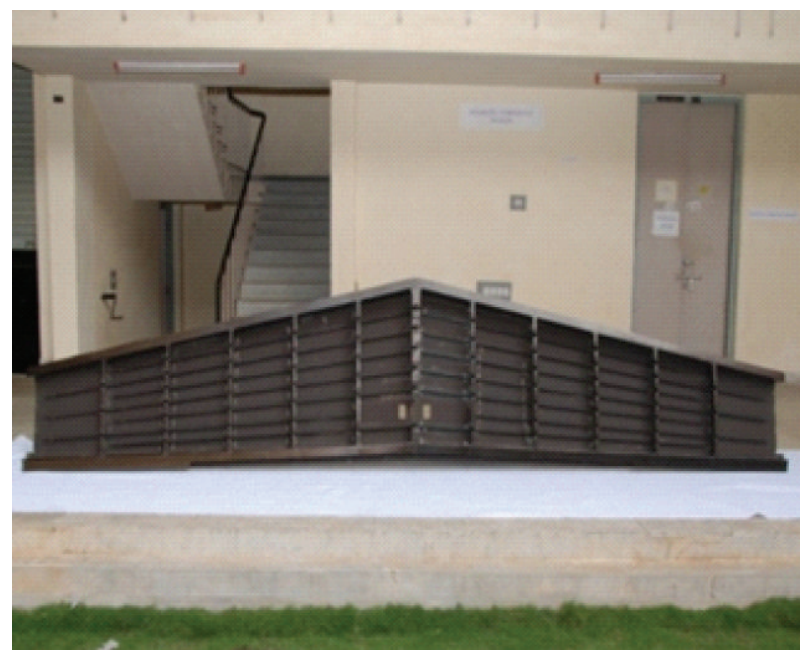

FIGURE 14: HT of SARAS aircraft, which was cured in an autoclave at CSIR-NAL.

not need frequent access during operation, have been housed in the basement tier, that is, inside the autoclave pit. The systems that require more frequent access during operation of autoclave such as vacuum line instrumentation system components and sensors are provided on the floor level. Pressurization-system pipelines and associated valves were housed on the top of the autoclave shell as they require less frequent access, that is, only during scheduled maintenance.

Every system requires certain standard checks and tests before commissioning. For example, all the pressure lines need to be hydrotested and assembled before connecting to the autoclave. It is a standard practice to design the vacuum system pipelines and resin traps for autoclave pressure as these lines may get exposed to higher pressure during vacuum-bag failure. Flushing of all the pipelines is required so that no dirt, weld particles are left out. In the vacuum lines, it is essential that no water or moisture is left in the

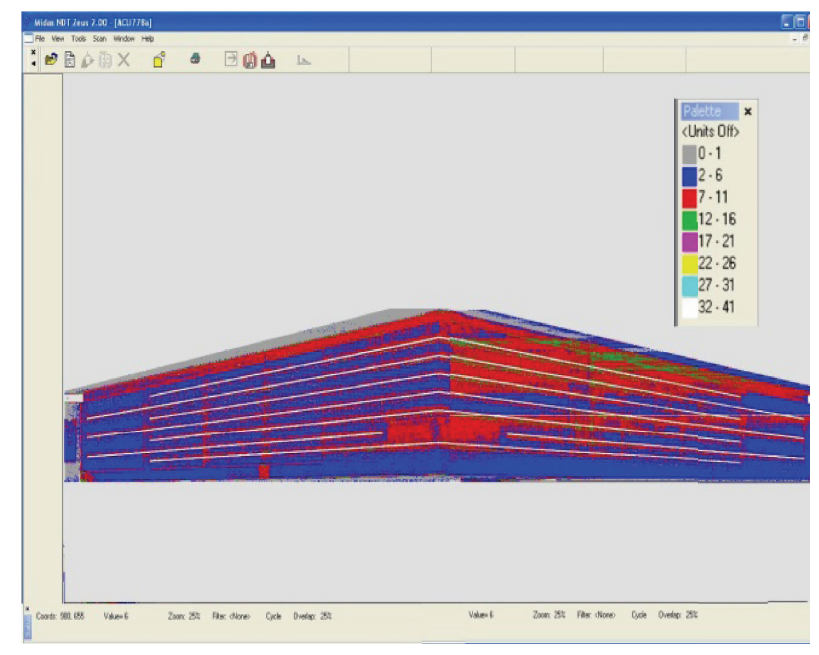

FIGURE 15: Screenshot of results of C-scan test conducted on HT of SARAS aircraft.

pipelines, as the moisture is detrimental to vacuum pump's performance. It is necessary to ensure that all the feedthroughs are pressure-tight.

A very exhaustive procedure is adapted to commission the autoclave. A detailed acceptance test plan (ATP), which is prepared well in advance, serves as the basis for commissioning. In addition, the manufacturer carries out elaborate tests to ensure proper operation. Every instrument, controller, sensor, and other devices are tested for their respective operations as per their specifications before their integration with other systems. The control system is divided into functional subgroups, such as data acquisition system, sequence and interlock control system, safety system, temperature, pressure, and vacuum control system and tested independently. Required accuracy levels are achieved in the control of temperature, pressure and vacuum through system tuning and repeated trials. The temperature uniformity in the autoclave is established with both full charge and no charge under all operating conditions. In order to ensure the ability of the system to achieve the maximum rate of heating and cooling, thermal insulation levels at worst-case situations, under maximum charge are established. Cure trials are carried out in a systematic manner, starting from typical composites test coupons and concluding with the curing of large composites structures. A number of cure trials depicting various cure cycles on various sizes of components are carried out. The cured parts are subjected to Non destructive testing and mechanical testing to ascertain proper curing. The tests and results are well documented for future reference. The test results of a specimen are given in Table 1 .

Figures 13 and 14 show the trouser duct of LCA aircraft and the horizontal tail (HT) of SARAS aircraft, which were cured in autoclaves at CSIR-NAL. Figure 15 shows the screenshot of results of C-scan test conducted on HT of SARAS aircraft.

The proper integration of all the sub-systems described above such that they eventually function as one entity is the key to successful realization of these complex equipments. 
TABLE 1: Test report of a sample composite specimen. Material: Hexply G0827 BB1040 HP03 1F; Resin Epolam 2063.

\begin{tabular}{|c|c|c|c|c|c|c|}
\hline \multicolumn{7}{|c|}{ Interlaminar shear strength (ILSS) at room temperature } \\
\hline Serial. no. & Width $(\mathrm{mm})$ & Thickness (mm) & Max. Load $(\mathrm{N})$ & ILSS (MPa) & Min. req. value (Mpa) & Failure mode \\
\hline$(1)$ & 10.06 & 1.97 & 1864 & 70.54 & & $\mathrm{~B}$ \\
\hline (2) & 10.1 & 1.92 & 1817 & 70.27 & Min: 60 & B \\
\hline (3) & 10.03 & 1.94 & 1854 & 71.46 & Mean: 65 & B \\
\hline (4) & 10.05 & 1.91 & 1778 & 69.46 & Mean Obtained. 7062 & B \\
\hline (5) & 10.1 & 1.97 & 1893 & 71.36 & Mean Udained: /0.02 & B \\
\hline \multicolumn{7}{|c|}{ Flexural strength (FL. Str) at room temperature } \\
\hline Sl. No. & Width $(\mathrm{mm})$ & Thickness $(\mathrm{mm})$ & Max. Load (N) & Fl. Str $(\mathrm{MPa})$ & Min req. value (Mpa) & Failure mode \\
\hline (1) & 10.07 & 1.94 & 446.91 & 1415.5 & & A \\
\hline (2) & 9.91 & 1.92 & 446.91 & 1468.1 & Mean: 1300 & A \\
\hline (3) & 10.01 & 1.94 & 478.68 & 1518.3 & Mean Obtained. 144458 & A \\
\hline (4) & 10.05 & 1.98 & 460.04 & 1401.1 & 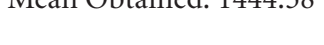 & A \\
\hline (5) & 10.02 & 1.95 & 450.85 & 1419.9 & & A \\
\hline
\end{tabular}

\section{Acknowledgments}

The authors would like to acknowledge the former Heads of Advanced Composites Division (ACD, CSIR-NAL), Mr. M. Subba Rao and Dr. M. R. Madhava, Head of ACD, Mr. H. N. Sudheendra, Head-ELK, Mr. M. V Krishna, Mr. M. C. Mahadeva, and Mr. Basuvaraj, ELK, Mr. K. V. Konda Reddy and Mr. T. V. Nataraj, EBU, Mr. K. Prakash and Mr. Amit Kumar Gupta, Mr. Partiban, and Mr. Palani of ACD for their contributions in developing the autoclave technologies.

\section{References}

[1] G. N. Dayananda, M. Subba Rao, and B. R. Somashekar, "Indigenous development of autoclave technology," in Proceedings of the National Symposium on Development in Advanced Composites and Structures, 1994.

[2] S. G. Advani and E. M. Sozer, "Processing advanced thermo set fiber composites," in Process Modeling in Composites Manufacturing, chapter 8, pp. 339-343, 2002.

[3] M. R. Monaghan and P. J. Mallon, "Development of a computer controlled autoclave for forming thermoplastic composites," Composites Manufacturing, vol. 1, no. 1, pp. 8-14, 1990.

[4] P. F. Stratton and D. G. Groome, "BOC gases, enhanced safety in advanced polymer composite curing autoclaves," in Proceedings of the 42nd International SAMPE Symposium and Exhibition, pp. 172-180, May 1997.

[5] G. M. Kamalakannan and M. Subba Rao, "Development of a computer based process control system for an autoclave to cure polymer matrix composites," in Proceedings of the International Conference on Instrumentation, Pune, India, December 2004.

[6] T. Taricco, "Autoclave cure systems," in ASM Engineering Hand book, Manufacturing Processes-Aerospace, 1991.

[7] Ramaswamy, Dayananda, and Subba Rao, "Development of a door steering mechanism for a large autoclave," in Proceedings of the National Conference on Recent Advances in Design Engineering ( $\left.R A D E^{\prime} 07\right), 2007$.
[8] G. M. Kamalakannan and A. K. Gupta, "An improvedtechnique and its implementation for control of high power heaters in large autoclaves and similar plants," Journal of the Instrument Soceity of India. In press.

[9] G. M. Kamalakannan, "Ethernet based online process monitoring and controlling of a plant having RS-232 or RS485 equipment," in Proceedings of the National Symposium on Instrumentation, NSI-32, Erode, India, October 2007. 

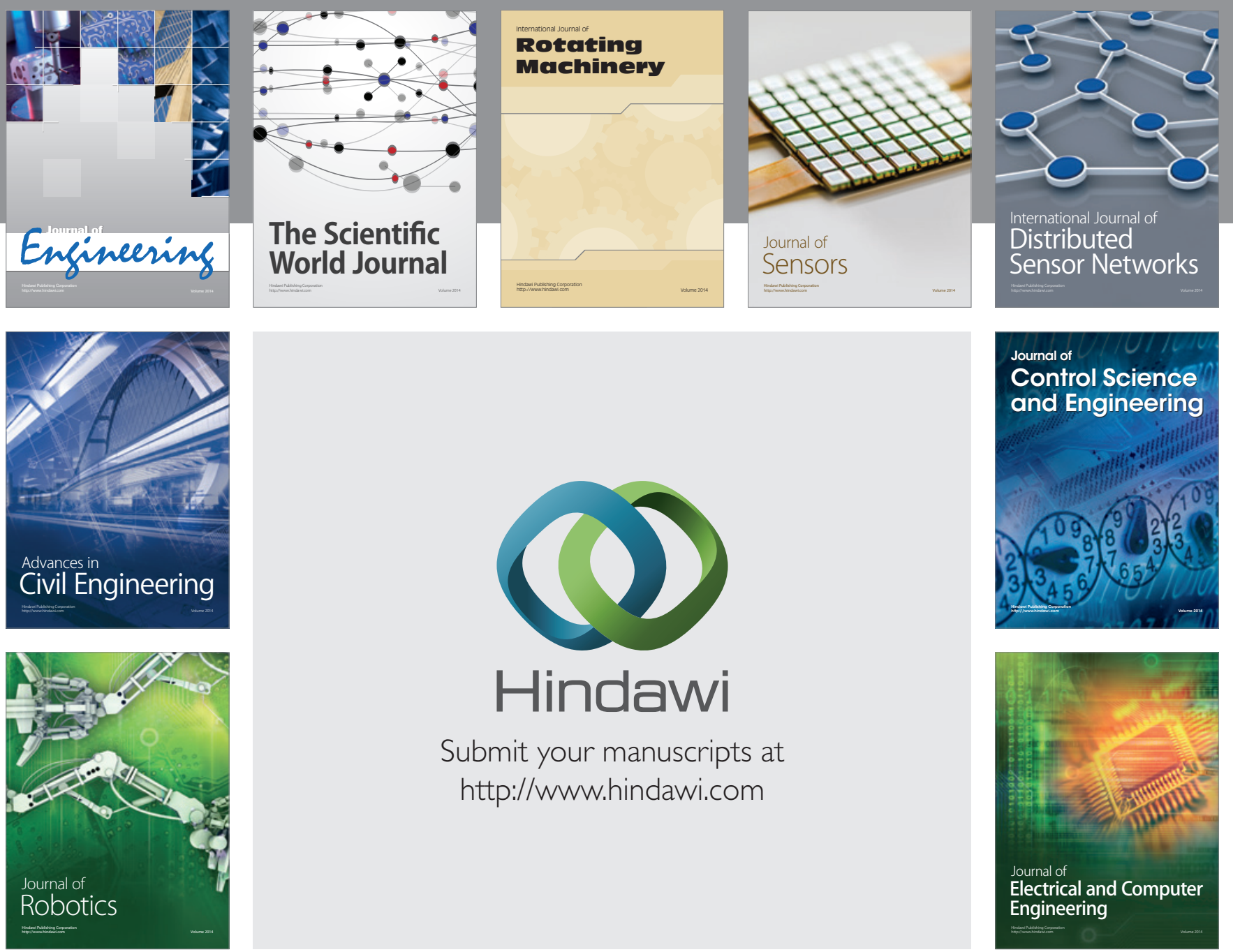

Submit your manuscripts at

http://www.hindawi.com
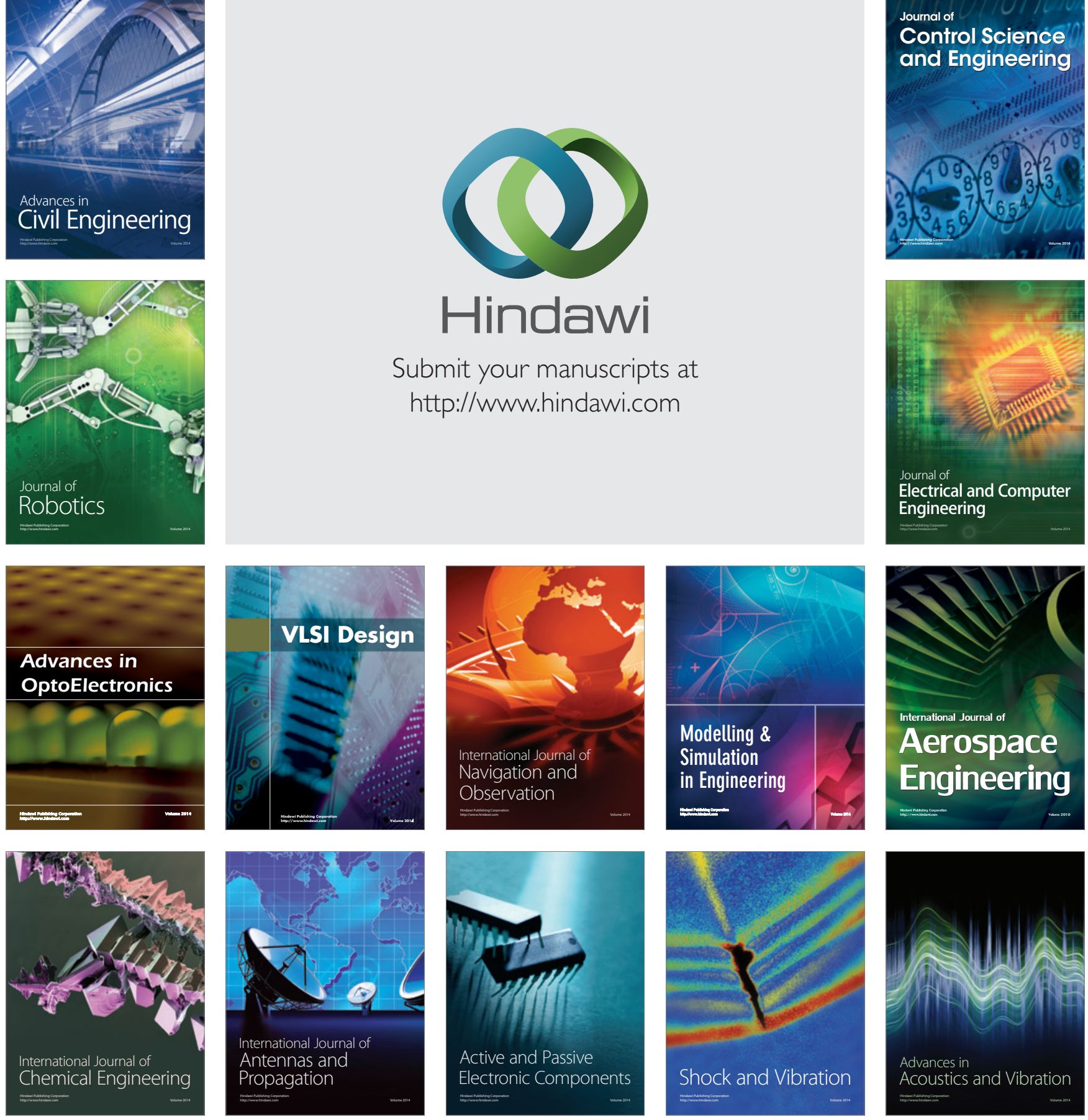